\title{
ADAPTIVE SYSTEM FORMING EXTREMELY HIGH SPEED OF MULTILINK MANIPULATOR GRIPPER
}

\author{
FILARETOV, V[ladimir] F[edorovich] \& GUBANKOV, A[nton] S[ergeevich]
}

\begin{abstract}
In a paper the forming method of maximum speed on a space trajectory of multilink manipulator gripper is offered. It takes into account restrictions of current and input voltage of actuators. Various modes of work of the synthesized system are researched at spatial trajectories having various curvatures.

Keywords: maximum velocity, multilink manipulator, restriction, adaptive control system, accuracy
\end{abstract}

\section{INTRODUCTION}

To improve performance of robotic equipment containing multilink manipulators (MM) it is necessary that all manufacturing operations performed with extremely high speeds, but the quality of the operations must be at the required level. At present a number of control systems (CS) synthesis methods are developed [1-3]. To improve the dynamic control accurasy these CS (combined, robust, adaptive, self-tuning) use highly sophisticated regulators including global, local, and adaptive control channels. In addition, these CS can provide high dynamic accuracy when moving fast in all areas of complex spatial trajectories (especially with high curvature) only under the condition that all actuators have sufficient capacity resource, i.e. they able to work out any input signal and simultaneously compensate for the negative effects arising from the dynamic interaction between all the degrees of freedom without entering saturation. If at least one of the MM actuators enters saturation the MM working tool (WT) goes out defined trajectory leading to a reject or even of an emergency. To solve this situation conventional $\mathrm{CS}$ has to reduce the velocity of the MM WT reducing the performance of it work, even when all it actuators have significant reserve power for control.

In [4] the synthesis method of time optimal DC drive with power restriction are considered. However, the use of this method for MM causes difficulties in obtaining analytically required control laws due to complex nonlinear transformations between joint and absolute coordinate system in which the gripper moves. Moreover, according to the authors, they obtained control law provides the minimum of transient time only if input signals are steps, which are not used for MM path control.

In [5] also is considered the time optimal CS MM providing the movement of WT along the desired trajectory with the maximum velocity determined by the kinematics of the MM and trajectory type. However, the high complexity of this method does not seem to allow for efficient use of real-time for complex MM with many degrees of mobility greater than two.
In [6] a CS synthesis method for motion mode of MM which automatically generates a maximum current speed of its movement along a path with a given dynamic accuracy are considered. However, the system can take into account only indirectly possible entry into saturation of individual actuators MM and definition of WT deviations from the path is difficult.

Thus, the task of forming speed MM WT along complex path to improve the performance of its work with the possible entry of actuators at saturation is actual and still unresolved.

\section{TASK SETTING}

The goal of this paper is the synthesis and study of adaptive formation of the maximum possible speed of the MM WT along a given spatial trajectory with the restriction for current and voltage of the input signals of all its actuators.

\section{DESCRIPTION OF ADAPTIVE SYSTEM FORMING EXTREMELY HIGH SPEED OF MULTILINK MANIPULATOR GRIPPER}

As stated above, the main task is to develop adaptive CS providing extremely fast movement of MM WT along given trajectory at which none of the actuators goes into saturation zone or significant nonlinearity and saving a high dynamic accuracy of control. That is this extremely fast MM motion on any space trajectory by means of special CSshould be supported such that one or more of its actuators will be constantly on the ending of their linear zones. In this case, the dynamic accurasy of the MM movement will be determined by the quality of each actuator CS, working in their linear zones.

On Fig. 1 shows a generalized MM control scheme containing formation speed loop of WT at the given trajectory and CS of each MM degree of mobility.

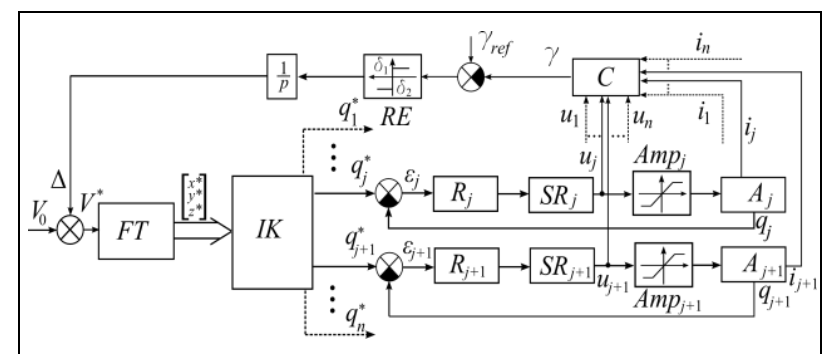

Fig. 1.The scheme of adaptive system forming extremely possible WT speed of motion 
On this figure: $V_{0}, V^{*}$-initial and formed WTmotion speeds accordingly; $F T$ - forming of WT trajectory taking into account current value $V^{*} ; x^{*}, y^{*}, z^{*}$ - program values of WT Cartesian coordinates; $I K$ - inverse of kinematics problem solution, forming input signals $q_{j}^{*}$ for all actuators $A_{j}$ of $\mathrm{MM} ; S R_{j}$ - self-turning regulators; $R_{j^{-}}$ conventional regulators; $A m p_{j}$ - amplifiers; $p$ differentiation symbol; $q_{j}$ - MM generalized coordinates; $\varepsilon_{j}$ - errors of tracking actuators; $u_{j}$ - inputs signals of corresponding amplifiers; $i_{j}$ - anchor currents of actuators; $C$ - criterion selection; $\gamma$ - dimensionless signal on $C$ exit; $\delta_{1}, \delta_{2}$ - threshold values of relay element $R E ; \Delta$ magnitude of velocity increment; $\gamma_{\text {ref }}$ - reference value; $j=\overline{1, n} ; n$ - number of axes of MM.

When WT moving along given spatial trajectory permissible value of the resulting dynamic error is provided by introduction to the straight chain of each actuator two regulators $R_{j}$ and $S R_{j}$ taking into account that all these actuators work in their linear zone without an input in saturation. Thus self-tuning regulators $\mathrm{SR}_{\mathrm{j}}$ stabilize parameters at nominal level [1] and typical regulators $P_{j}$, which parameters are calculated by conventional methods, provide the required dynamic accuracy.

While all actuators of MM work in a linear zone, the condition $\left|\gamma_{\text {ref }}\right\rangle|\gamma|$ is satisfied, and the difference $\gamma_{\text {ref }}-\gamma$ is positive. In this case according to the figure $1 \mathrm{WT}$ velocity will increase with a constant acceleration $\delta_{1}$. If some input signals of $C\left(u_{j}\right.$ or $\left.i_{j}\right)$ override threshold behind which begin significant nonlinearities zones of actuators or even a zone of their saturation the condition $\left|\gamma_{\text {ref }}\right|<|\gamma|$ starts to be satisfied, and the difference $\gamma_{\text {ref }}-\gamma$ becomes negative. In this case WT velocity on a trajectory will be reduced with constant acceleration $\delta_{2}$, raising thereby a dynamic accuracy of control. Values $\delta_{1}$ and $\delta_{2}$ depend on dynamic properties of MM actuators and also from used CS and selected by means of simulation.

Studies have shown that for all actuators in their linear zone when WT moving along path with small curvature is sufficient to form $\gamma$ (Fig. 1) as:

$$
\gamma=\max \left(\left|u_{1}\right|, \ldots,\left|u_{j}\right|, \ldots,\left|u_{n}\right|\right),
$$

that is use the information only about values of voltages $u_{1}, \ldots, u_{n}$ inputted to the respective amplifiers. These results in the selection of the most loaded MM actuator and determined the maximum possible speed of the WT maintaining given level of a dynamic error. If the spatial trajectory has a significant curvature, the signal $\gamma$ should be formed on the basis of measurements of currents $i_{1}, \ldots, i_{n}$ in the anchor chains of all electric motors

$$
\gamma=\max \left(\left|i_{1}\right|, \ldots,\left|i_{j}\right|, \ldots,\left|i_{n}\right|\right) .
$$

These results from the fact that the current of an anchor $i_{j}$ unlike voltage $u_{j}$ is more informative signal because it is formed taking into account back emf and is proportional to rotational speed of the actuator. In addition, it is proportional to developing torque and hence the acceleration of the rotor of the electric motor. When using expression (2) the system begins to rapidly reduce WT speed approaching the path sections with a large curvature. Value of curvature of individual sections of the trajectory, which determines use of (1) or (2) depends on the kinematic characteristics of the MM and the requirements for dynamic accuracy of WT motion.

However, studies have shown that there are a large number of trajectories to support the work of all actuators in their linear zones use the information only for voltages $u_{1}, \ldots, u_{n}$ or currents $i_{1}, \ldots, i_{n}$ only is not enough. In this case, the signal $\gamma$ should be formed under the law:

$$
\begin{aligned}
\gamma= & K_{1} \max \left(\left|u_{1}\right|, \ldots,\left|u_{j}\right|, \ldots,\left|u_{n}\right|\right)+ \\
& +K_{2} \max \left(\left|i_{1}\right|, \ldots,\left|i_{j}\right|, \ldots,\left|i_{n}\right|\right),
\end{aligned}
$$

where $K_{1}$ and $K_{2}$ - some positive weights whose values are determined in the simulation or experiment. The studies showed that the choice of $\gamma$ in the form of (1) or (2) the value $\gamma_{\text {ref }}$ usually is selected approximately $20 \%$ less than the maximum allowable value $\left|u_{j}\right|$ or $\left|i_{j}\right|$ in which work of all actuators still is carried out in their linear zones $(j=\overline{1, n})$. When use $\gamma$ in the form (3) the $\gamma_{r e f}$ can be reduced by $20-30 \%$ compared with the specified maximum permissible values $\left|u_{j}\right|$ or $\left|i_{j}\right|$.

Thus, the developed adaptive system (see fig. 1) should provide the WT movement along any smooth paths on extremely possible speed with given dynamic accuracy which is achieved by means of standard regulators due to the formation of such program signals which provides hard work of one or more of the actuators, keeping these and other electric motors in linear zones without input in saturation. This approach can be use for any industrial manipulators.

\section{STUDY OF ADAPTIVE SYSTEM FORMING EXTREMELY POSSIBLE SPEED OF MULTILINK MANIPULATOR GRIPPER}

Research of developed adaptive system was conducted using PUMA MM shown in fig. 2. Only its portable degrees of mobility were considered. Here we use the following notation and set the following parameters: Oxyz- Cartesian coordinate system; $S$ trajectory; $l_{1}=l_{2}=l_{3}=0.5 \mathrm{~m}$ - lengths of MM links; $m_{g}$ $=5 \mathrm{~kg}, m_{1}=25 \mathrm{~kg}, m_{2}=m_{3}=5 \mathrm{~kg}-$ accordingly, masses of load and links; $J_{s 1}=0.1 \mathrm{~kg} \cdot \mathrm{m}^{2}, J_{s 2}=0.007 \mathrm{~kg} \cdot \mathrm{m}^{2}, J_{s 3}=$ $0.005 \mathrm{~kg} \cdot \mathrm{m}^{2}$ - moments of inertia of these links concerning their centerlines; $J_{n 2}=0.14 \mathrm{~kg} \cdot \mathrm{m}^{2}, J_{n 3}=0.2$ $\mathrm{kg} \cdot \mathrm{m}^{2}$ - moments of inertia of corresponding links concerning their centres of masses, perpendicular to centerlines.

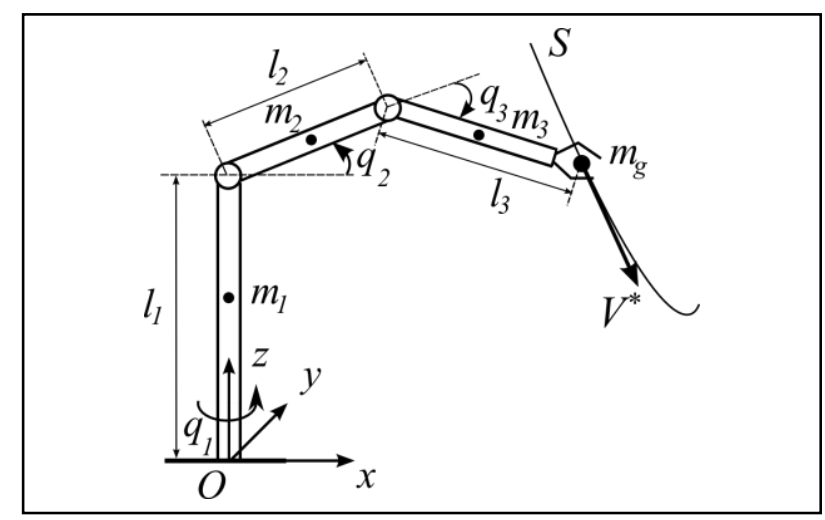

Fig. 2. Kinematic scheme of PUMA manipulator 
As actuators MM were selected three identical DC motor with the following parameters: $R=0.5 \Omega$ resistance of an anchor chain; $L=0.0005 \mathrm{H}$ - inductance of an anchor chain; $K_{\omega}=0.04 \mathrm{~V} \cdot \mathrm{s}-$ emfcoefficient; $K_{m}=$ $0.04 \mathrm{~N} \cdot \mathrm{m} / \mathrm{A}$ - torque coefficient; $J=10^{-3} \mathrm{~kg} \cdot \mathrm{m}^{2}$ moment of inertia of the rotating parts of the motor and gear unit; $i_{p}=100$ - gear ratios; $K_{y}=800$ gain coefficient; $u_{\max }= \pm 27 \mathrm{~V}-$ limit of input voltage. In system on fig. $1 \delta_{1}$ $=1, \delta_{2}=-1$.

Transfer functions of all regulators $R_{j} j=\overline{1,3}$ ) are as follows:

$$
W_{p j}(p)=\frac{(0.128 p+1)(0.189 p+1)}{(0.0125 p+1)(0.87 p+1)}
$$

On fig.in 3 numbers 1 and 2 denote the trajectory described by the expressions $y(x)=0.15 \sin (2 x)+0.1$ and $y(x)=0.15 \sin (14 x)+0.1$ accordingly, and by number 3 - a circular trajectory with radius $0.15 \mathrm{~m}$ on which WT moves (see fig. 2). MM base has coordinates $O(0,0)$.

First we will consider WT motion along trajectory with small curvature (see a curve 1 on fig. 3 ) when the signal $\gamma$ is formed with use of expression (1), and $\gamma_{\text {ref }}=$ $21 \mathrm{~V}$. Figure 4 shows that all time one of MM actuator is on the ending of its linear zone, providing the maximum speed of the WT: in the beginning the first (see $u_{1}$ ), then the second (see $u_{2}$ ) and at last the third (see $u_{3}$ ). Figure 5 shows the processes of changing $V^{*}, y^{*}, x^{*}$ as WT moves along the path 1 . Here and below we use the following scales: $V^{*}=$ scale $1 \mathrm{~m} / \mathrm{s} ; y^{*}=$ scale $1 \mathrm{~m} ; x^{*}=$ scale $1 \mathrm{~m} ; \varepsilon_{d}=$ scale $0.0025 \mathrm{~m}$. The velocity $V^{*}$ along this trajectory initially increases up to $1.1 \mathrm{~m} / \mathrm{s}$ and then decreases for maintaining the required dynamic error level, which does not exceed $2 \mathrm{~mm}$. Thus the deviation from trajectory of theWT does not exceed $1 \mathrm{~mm}$. Maximum constant speed at which the MM WT can follow trajectory 1 without sharp increase of the dynamic error exceeding $2 \mathrm{~mm}$, by the entrance of its actuators into saturation is only $0.37 \mathrm{~m} / \mathrm{s}$. As a result designed adaptive system forming a signal $\gamma$ according to expression (1) allows for 1.96 times to reduce execution time of the movement.

On figures 6 and 7 currents in MM DC motors and laws of changing $V^{*}, y^{*}, x^{*}, \varepsilon_{d}$ are shown at WT motion on the same trajectory 1 , but the formation of the signal $\gamma$ is in accordance with the expression (2).

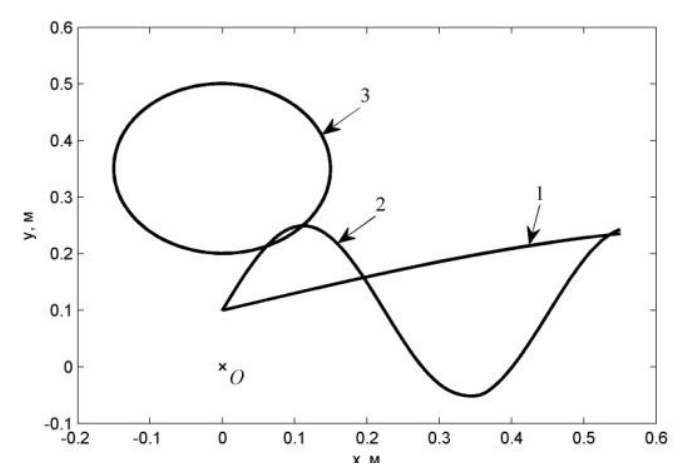

Fig. 3. WT motion trajectories

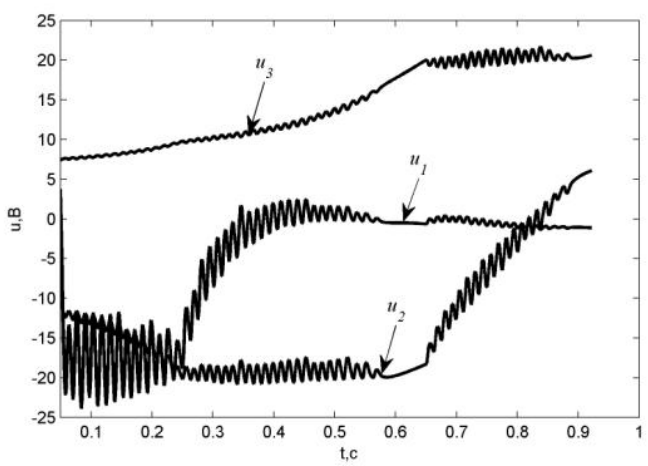

Fig. 4. Input voltages of manipulator actuators

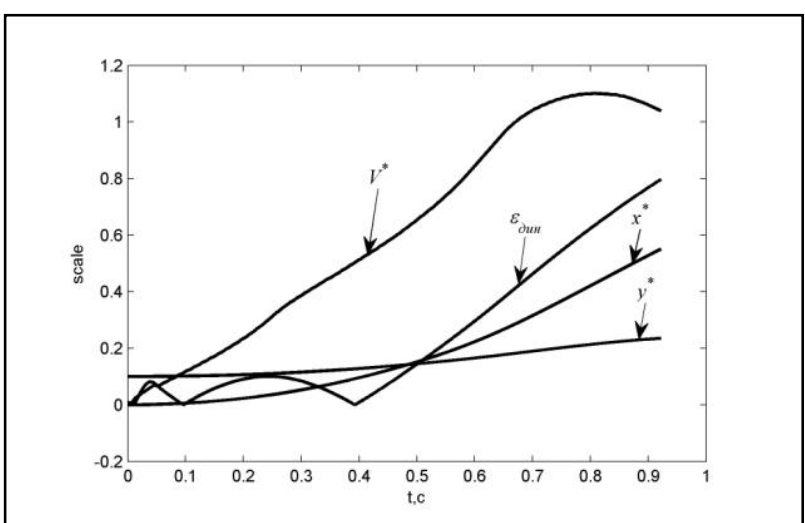

Fig. 5. Processes of changing $V^{*}, y^{*}, x^{*}, \varepsilon_{d}$ when $\gamma$ determining from (1)

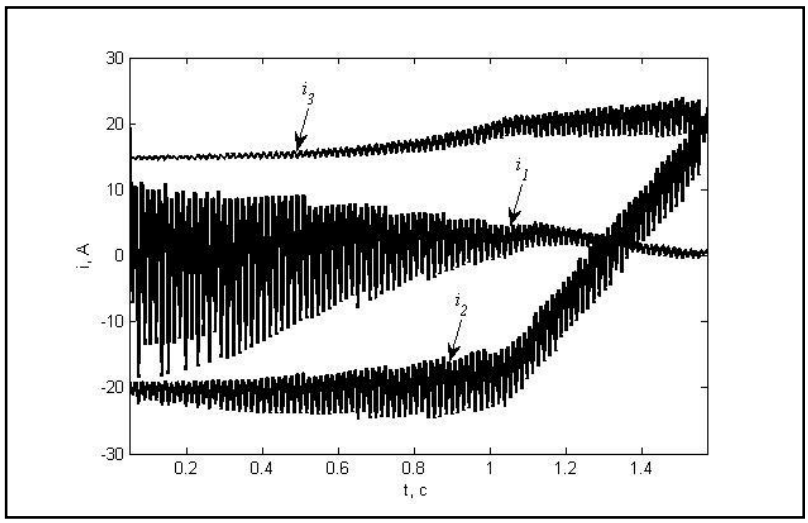

Fig. 6. Currentsin MM actuators

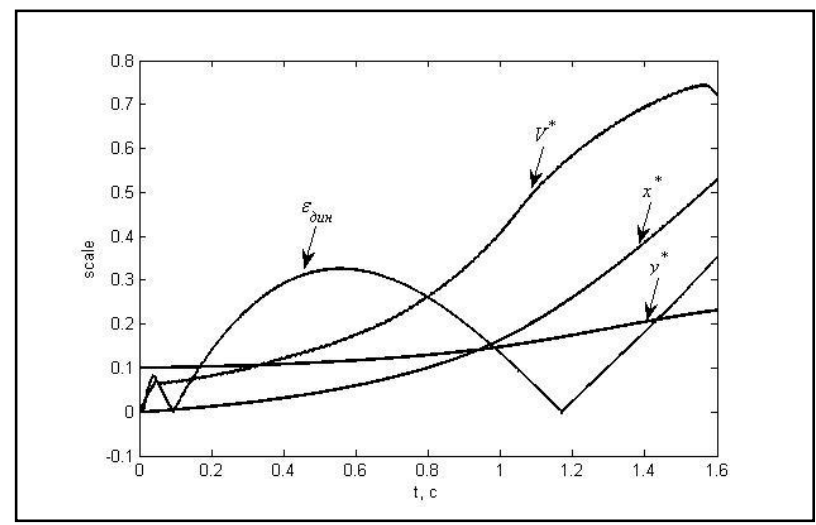

Fig. 7. Processes of changing $V^{*}, y^{*}, x^{*}, \varepsilon_{d}$ when $\gamma$ determining from (2)

Figure 6 shows that the currents in all MM motors does not exceed a reference value $\gamma_{r e f}=20 \mathrm{~A}$. To the end of its linear zone first come the second $i_{2}$ and then the 
third $i_{3}$ motor. High-frequency oscillations of currents created by the use of $S R_{j}$. Fig. 7 shows that in this system

the maximum speed of WT becomes 1.47 times less than in the first system and the total run time of the whole movement - 1.68 times more. However, the value of $\varepsilon_{d}$ rather than the previous system does not exceed $0.8 \mathrm{~mm}$.

Reducing WT speed at forming $\gamma$ in accordance with (2) is due to that the current signal contains information about the exretnal torque on each actuator and it increases (in this example for the second actuator) as the "telescoping" of MM (see path 1 in Fig. 3).

Figures 8 and 9 shows the currents in the MM motors and processes of change in $V^{*}, y^{*}, x^{*}, \varepsilon_{d}$ at WT motion along path 2 (see Fig. 3), when $\gamma$ is determined by the expression (2). Figure 8 shows that the adaptive system by tuning the speed maintains one of the actuators at the end of its linear zone. Momentary peaks of currents due to the presence of recurvatures in the trajectory and as a consequence velocity sign changings in any degrees of mobility. However, for DC motors short two-times excess of the nominal value of the current is quite acceptable. The maximum value $\varepsilon_{d}$ for the complex motion of the WT is less than $3 \mathrm{~mm}$ at $0.6 \mathrm{~m} / \mathrm{s}$ speed.

When WT moved along harmonic trajectory 2 (see Figure 3), but $\gamma$ is formed according to (3)there was almost the same level of $\varepsilon_{d}$ taking into account the actuator input voltage increases the maximum speed of the WT in 1.37 times that in comparison with earlier considered system which is tuned only on currents, raises productivity of operation of MM on $25 \%$. Integrally, in comparison with the system working from constant $V^{*}$ and with the same limiting value $\varepsilon_{d}$ the movement along the path 2 is reduced by 2.4 times.

Figure 10 shows the processes of change in $V^{*}, y^{*}$, $x^{*}, \varepsilon_{d}$ when WT moving along circular path 3 (Fig. 3) in the adaptive system (see fig. 1) when $\gamma$ is set according (3). This system, as well as in the previous case, works on $27 \%$ faster the system using signal $\gamma$ in form (2). And its performance is increased 2.55 times compared with the system operating at a constant speed.

It should be noted that due to the choice of parameters $\gamma_{r e f}, K_{1}$ and $K_{2}$ can achieve different combinations of $V^{*}$ and $\varepsilon_{d}$. Furthermore decrease of $\varepsilon_{d}$ can be achieved through the introduction of electric servo systems of MM better regulators.

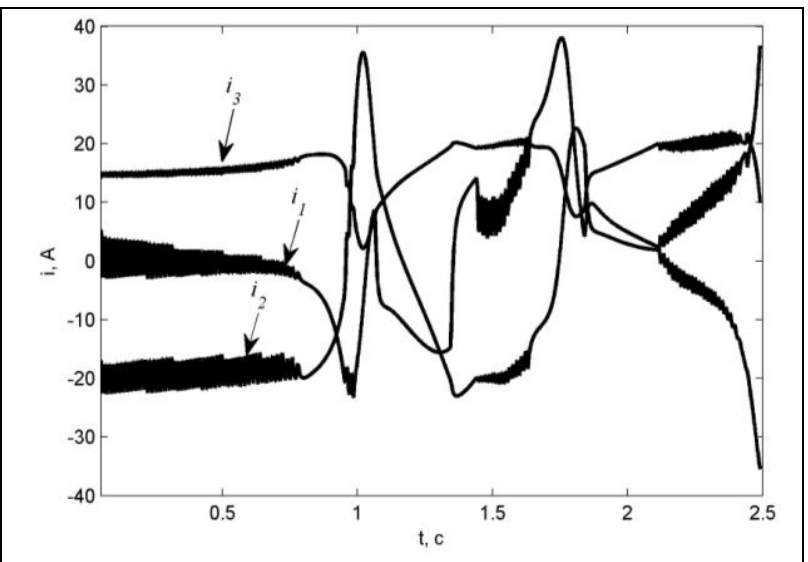

Fig. 8. Currents in manipulator actuators

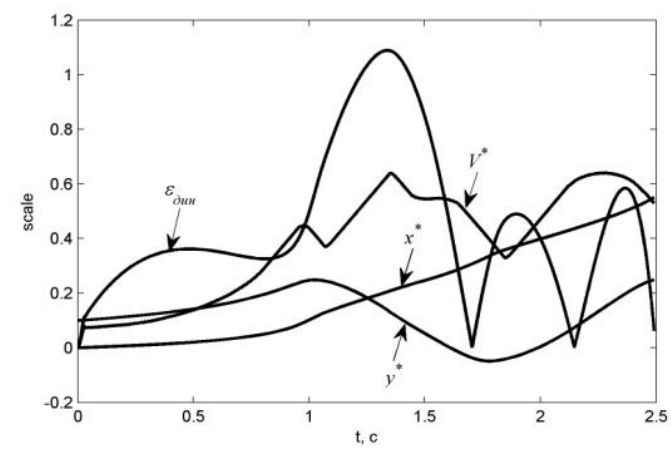

Fig. 9. Processes of changing $V^{*}, y^{*}, x^{*}, \varepsilon_{d}$ when $\gamma$ at definition $\gamma$ according to (2)

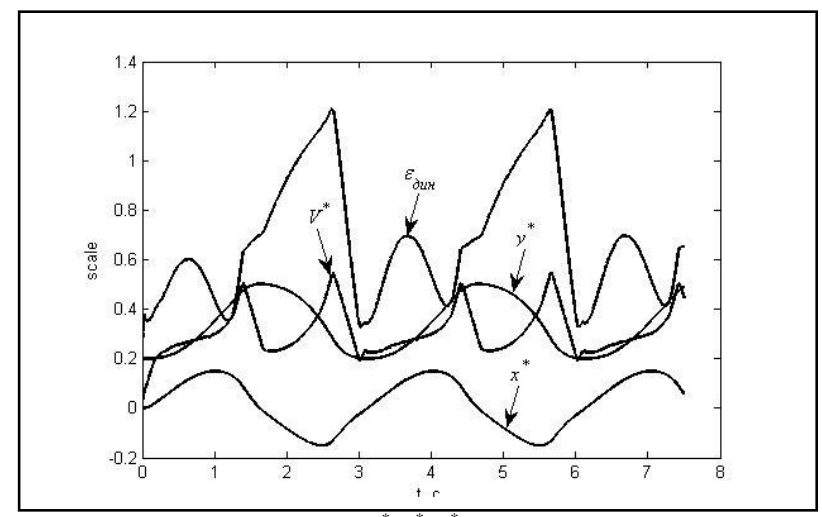

Fig. 10. Processes of changing $V^{*}, y^{*}, x^{*}, \varepsilon_{d}$ when $\gamma$ at definition $\gamma$ according to (2)

\section{CONCLUSION}

In this papera system, which provides the maximum possible speed of the MM WT along arbitrary spatial trajectories, based on the information about the input voltage and current in MM actuators is considered. The above system does not allow entry of these actuators in the saturation regime leading to a sharp reduction of the dynamic accuracy of control. Furthermore one can essentially improve the performance of manufacturing operations performed by MM.

\section{ACKNOWLEDGEMENTS}

This paper is supported by RFBR (Grants № 11-0798502, 11-08-98505) and Federal target program "Scientific and educational personnel of innovative Russia " 2009-2013.

\section{REFERENCES}

[1]. Filaretov, V.F. (2000). Self-tuning control system of actuators of manipulators, FENTU, Vladivostok, Russia

[2]. Siciliano, B.\&Khatib, O. (2008). Handbook of robotics, Springer Verlag, Berlin

[3]. Zenkevich, S.L. \&Yushenko, A.S. (2004). The basis of manipulator robots control, BMSTU Press, Moscow. (in Russian)

[4]. Morzhov, A.V. \&Faldin, N.V. (2011) Synthesis of time-optimal tracking dc electric drive with limitation on consumed power. Journal of computer and systems sciences international, Vol. 50. No. 2., 205-219, 1064-2307

[5]. Pshikhopov,V.Kh. (2007) Time-optimal path control for robot manipulator. Izvestiyavuzov. Elektromehanika, No. 1, 51-57, 01363360. (in Russian)

[6]. Filaretov, V. F.; Yukhimets, D. A. \&Konoplin A. Yu. (2012)Method of Synthesis of System of Automatic Control of Manipulator's Gripper Movement Mode on Difficult Spatial Trajectories.Mechatronics, automatization and control, No. 6, 4754, 1684-6427. (in Russian) 